\title{
Motion Flow Segmentation Based on Optical Flow Angle
}

\author{
Kun Yang, Lin Wang \\ Guizhou Minzu University \\ Guiyang, China \\ e-mail:wanglin@gzmu.edu.cn
}

\author{
Fu-Jian Feng, Jiang-Hao Yu, Yuan-Fei Cheng \\ Guizhou Minzu University \\ Guiyang, China \\ e-mail: kunflv@126.com
}

\begin{abstract}
Aiming at the irrationality of deep motion state analysis, such as density estimation, target tracking and behavior understanding, which taken the motion foreground as a whole in dense crowd scene. This paper proposes an algorithm to segment motion flow based on optical flow angle. First of all, the motion prospect is obtained by using the finite-time Lyapunov exponent (FTLE) algorithm. Then get the foreground optical flow information with the prospect as a mask. Finally, the dynamic K-means clustering method is applied to segment the foreground optical flow angle. Experimental results show that the proposed algorithm is clear and easy to implement, and the motion flow can be segmented accurately in different scenes.
\end{abstract}

Keywords-Optical flow; Lyapunov exponent; Crowd target detection; Motion flow segmentation.

\section{INTRODUCTION}

With the economic and social development, many events of people gathered are increasing gradually, such as sports, concerts, religious meetings and so on. Due to different crowed flow with different speed, direction and local crowd density, as well as the objective constraint that the carrying capacity of the road is limited and other factors in similar scenes. Making it is possible, which the crowd prone to congestion and even stampede accident, resulting in loss of life and property in crowd incidents. In order to avoid accidents effectively in crowd incidents, intelligent monitoring technology has been used in stations, airports, subways and large shopping centers and other fields. while, it is the key step to analysis movement state that motion flows are segmented reasonably and effectively in the intelligent monitoring technology. It is also a research focus on computer vision and image processing.

There may be multiple motion flows in scene of crowd event, and there are obvious differences in velocity, direction of motion, and crowd density between motion flows. It is unwise to estimate the crowd density, target tracking and behavior understanding, treating the motion prospect as a whole. Therefore, it is necessary to classify the motion foreground which has been extracted. The condition for estimating crowd density rapidly and monitoring state of crowd accurately is that we can detect the crowd efficiently. As well as the prepare condition to avoid accidents, which are caused by the crowd movement with abnormal state.

At present, the most commonly adapted methods for detecting objects of prospect in crowd incidents include global optical flow, background updating approach and fluid dynamic method [1][2]. Specifically, Davies [3] proposed a method by using the technology of image processing for crowd monitoring whose main idea is to analyze the size of crowd and estimate the crowd movement. R. Ma [4] etc. Proposed a density estimation algorithm, which is suitable for monitoring systems. The algorithm employed the number of prospect pixels as a feature to estimate crowd density. But it can not work well, when the objects of prospect were sheltered from each others. V.Rabaud and S. Belongie [8] proposed a single target segmentation method. Firstly, the Kanade-Lucas-Tomasi tracing method was used to detect the moving objects in the foreground, then a clustering method based on spatiotemporal filtering was used to classify similar quantities of feature. Each class represents the trajectory of a target.

In contrast to the above method, T.Schlogl [5] proposed a novel method for estimating the crowd target by dividing the motion flow, detecting the number of each movement flow, cumulating the number to get the target amount at last. A.B.Chan and Z.Liang [6]proposed a statistical methods which is based on dynamic texture analysis . Firstly, the hybrid dynamic texture method is employed to segment the motion flow with different directions [7]. Then a set of feature quantities including area, edge, perimeter and other pixel characteristics and uniformity, energy, entropy and other texture features, is extracted from each motion flow. By normalizing the eigenvectors, the Gaussian regression is applied to train the relationship between the eigenvectors and the number of people. Different from the above methods, S. Ali [2] introduced the theory of particle dynamics to process images.

As pointed out in [2], a structure similar to "ridge" appears in the FTLE [10] field, namely the Lagrangian coherent structure (LCS), as soon as there is a difference in motion between adjacent particles. Finally, the motion flow segmentation is realized by LCS. Because FTLE is a scalar field [6], even crowds with different motion directions will be classified into one kind of motion flow when the fluctuation range of FTLE is smaller. Aiming at the above analysis, a algorithm of motion flow segmentation based on optical flow angles is proposed.

\section{PRINCIPLES OF PARTICLE DYNAMICS}

\section{A. Dynamical Systems}

The general dynamical system of particle dynamics can be expressed as follows: 


$$
\left\{\begin{array}{l}
\dot{x}\left(t ; t_{0}, x_{0}\right)=\dot{v}\left(x\left(t ; t_{0}, x_{0}\right), t\right) \\
x\left(t ; t_{0}, x_{0}\right)=x
\end{array}\right.
$$

Where, $x\left(t ; t_{0}, x_{0}\right) \in D$ represents the current system state, $t$ is time, $\dot{x}\left(t ; t_{0}, x_{0}\right)$ represents the velocity function of the current state $x\left(t ; t_{0}, x_{0}\right)$ varying with time $t$, In general, $D$ is a subset of the n-dimensional space $R^{n}$. v(x $\left.\left(t ; t_{0}, x_{0}\right), t\right)$ can be understood as a time-dependent state of a vector function. The solution of (1) is stable, if the vector function is stable. The solution moves along with the trajectory of vector function $v\left(x\left(t ; t_{0}, x_{0}\right), t\right)$, when the time $t$ changes. That is to say, we get a set of particles containing different trajectories named flow map, when the time $t$ changes. If the initial time is $t_{0}$ and the ending time $t$, then we can get a flow map $\phi_{t_{0}}^{t}$ that represents the motion trajectory of a point $x$ from $t_{0}$ to $t$ :

$$
\phi_{t_{0}}^{t}: D \rightarrow D: x_{0} \mapsto \phi_{t_{0}}^{t}\left(x_{0}\right)=x\left(t ; t_{0}, x_{0}\right)
$$

Note that particle flow map has the properties as follows:

$$
\left\{\begin{array}{c}
\phi_{t_{0}}^{t}(x)=x \\
\phi_{t_{0}}^{t_{0}+s}(x)=\phi_{s}^{t+s}\left(\phi_{t_{0}}^{s}(x)\right)=\phi_{t}^{t+s}\left(\phi_{t}^{t}(x)\right)
\end{array}\right.
$$

We can obtain the numerical solution of (1) by integrating the value of $v\left(x\left(t ; t_{0}, x_{0}\right), t\right)$, when the vector function $v\left(x\left(t ; t_{0}, x_{0}\right), t\right)$ is time independent and is a linear function with respect to $x$. The crowd event motion system studied in this paper is time-dependent, therefore, it is necessary to find the fixed points and their adjacent points in the obtained vector field (optical flow field), and track the motion trajectories of these points to obtain the motion flow.

\section{B. The Lyapunov Exponent}

The Lyapunov exponent is an asymptotic quantity which measures the extent to which infinitely close particles separate in a finite amount of time. If the infinitely close particles $x$ and ${ }^{y}$ at the initial time ${ }^{t_{0}}$ satisfy the equation $y=x+\delta\left(t_{0}\right)$, and the $\delta\left(t_{0}\right)$ is the offset of $x$ and $y$ at the initial time,then the offset of $x$ and ${ }^{y}$ after time $t$ can be expressed as:

$$
\begin{aligned}
\delta\left(t_{0}+t\right) & =\phi_{t_{0}}^{t_{0}+t}(y)-\phi_{t_{0}}^{t_{0}}(x) \\
& =\frac{d \phi_{t_{0}}^{t_{0}+t}(x)}{d x}+o\left(\left\|\delta\left(t_{0}\right)\right\|^{2}\right)
\end{aligned}
$$

Where, $o\left(\left\|\delta\left(t_{0}\right)\right\|^{2}\right)$ is high-order infinitesimal, so it can be rounded off. Then the magnitude of the offset $\delta\left(t_{0}+t\right)$ is equal to:

$$
\begin{aligned}
& \left\|\delta\left(t_{0}+t\right)\right\|=\sqrt{\left\langle\frac{d \phi_{t_{0}}^{t_{0}+t}(x)}{d x} \delta\left(t_{0}\right), \frac{d \phi_{t_{0}}^{t_{0}+t}(x)}{d x} \delta\left(t_{0}\right)\right\rangle} \\
& =\sqrt{\left\langle\delta\left(t_{0}\right), \frac{d \phi_{t_{0}}^{t_{0}+t}(x)^{*}}{d x} \frac{d \phi_{t_{0}}^{t_{0}+t}(x)}{d x} \delta\left(t_{0}\right)\right\rangle}
\end{aligned}
$$

Where, $\Delta=\frac{d \phi_{t_{0}}^{t_{0}+t}(x)}{d x} \frac{d \phi_{t_{0}}^{t_{0}+t}(x)}{d x}$ is known as
Cauchy-Green strain tensor, while quantity $\frac{d \phi_{t_{0}}^{t_{0}+t}(x)}{d x}$ is the spatial gradient of the flow map, therefore (5) can be transformed into:

$$
\left\|\delta\left(t_{0}+t\right)\right\|=\sqrt{\left\langle\delta\left(t_{0}\right), \Delta \delta\left(t_{0}\right)\right\rangle}
$$

As can be seen from equation (6), the maximum offset between infinitesimally close particles occurs when $\delta\left(t_{0}+t\right)$ is aligned with the eigenvector of maximum eigenvalue of $\Delta$. It can be expressed as follows:

$$
\begin{aligned}
\max \left\|\delta\left(t_{0}+t\right)\right\|= & \sqrt{\left\langle\delta\left(t_{0}\right), \lambda_{\max }(\Delta) \delta\left(t_{0}\right)\right\rangle} \\
& =\sqrt{\lambda_{\text {max }}(\Delta)}\left\|\delta\left(t_{0}\right)\right\|
\end{aligned}
$$

In equation (7), $\lambda_{\max }(\Delta)$ is the maximum eigenvalue of $\Delta$.If equation (7) is defined [10] as follows: 


$$
\sigma_{t_{0}}^{t}(x)=\frac{1}{|t|} \ln \sqrt{\lambda_{\max }(\Delta)}
$$

Equation (8) is the computational expression of the finite time Lyapunov exponent [10]. When $\sigma>0, x$ and $y$ are divergent; When $\sigma=0, x_{\text {and }}{ }^{y}$ relative position remains unchanged; When $\sigma<0, x$ and $y$ are likely to aggregate together. Finally, prospect detection and motion flow segmentation are realized by LCS in FTLE field. Specific algorithm steps are as follows:

1) Calculate the global optical flow field, averaging 5 consecutive frames to eliminate the noise interference and storage;

2) Compute the particle flow map $\phi_{t_{0}}^{t}$ by using Linear interpolation;

3) We can employ the equation $\frac{d \phi_{i j}^{i+t}}{d x}=\left[\begin{array}{ll}\frac{x_{i+1, j}\left(t_{0}+t\right)-x_{i-1, j}\left(t_{0}+t\right)}{x_{i+1, j}(t)-x_{i-1, j}(t)} & \frac{x_{i+1, j}\left(t_{0}+t\right)-x_{i-1, j}\left(t_{0}+t\right)}{y_{i, j+1}(t)-y_{i, j-1}(t)} \\ \frac{y_{i+1, j}\left(t_{0}+t\right)-y_{i-1, j}\left(t_{0}+t\right)}{x_{i+1, j}(t)-x_{i-1, j}(t)} & \frac{y_{i+1, j}\left(t_{0}+t\right)-y_{i-1, j}\left(t_{0}+t\right)}{y_{i, j+1}(t)-y_{i, j-1}(t)}\end{array}\right]$

to calculate the spatial gradient, then the Cauchy-Green strain tensor is obtained by $\Delta=\frac{d \phi_{t_{0}}^{t_{0}+t}(x)^{*}}{d x} \frac{d \phi_{t_{0}}^{t_{0}+t}(x)}{d x}$;

4) The Lyapunov exponent is calculated using the equation (8). Using the location and correlation of "ridge" in FTLE field, the crowd target extraction and motion flow segmentation are realized.

\section{MOTION FLOW SEGMENTATION BASED ON OPTICAL FLOW ANGLE}

Generally speaking, different crowed flow with different speed, direction and local crowd density in the group event. Therefore, it will improve the segmentation effect of motion flow, if we can find a feature to depict the movement direction effectively in the process of motion flow segmentation. It reminds us of global optical flow, which includes the speed of all pixels, but also reflects the movement direction (optical flow angle). Therefore, we proposed a motion flow segmentation algorithm based on optical flow angle.

\section{A. Sample Acquisition and Initialization Clustering Parameters}

Considering the optical flow is calculated by the method of global optical flow [11], there is also optical flow value in the background. In order to eliminate the interference of the optical flow value in the background, we take the foreground obtained by the algorithm in [2] as a mask, extracting the corresponding angle information of the optical flow to form a sample space. Then carry out dynamic K-means clustering to them. The size of the sample space is the same as the image size and the range of the sample is $[0,2 \pi)$.

The number of samples is much larger than the number of clusters by observing the sample space and the actual scene. So a good result will be achieved depending on the algorithm of Dynamic K-means clustering [11]. Initialize the cluster center $C=\left[0, \frac{\pi}{2}, \pi, \frac{3}{2} \pi\right] \quad, \quad$ intra-class Tin $=\frac{\pi}{4}$, interclass thresholds $T b=\frac{\pi}{2}$ and the maximum number of cycles $t=10$.

B. Algorithm Steps

1)Initial clustering of samples: If $\left|\theta_{i}-C_{j}\right| \leq$ Tin, then $\theta_{i}$ belongs to the $j$ class;

2) Update the cluster center according to $C_{j}=\frac{1}{n} \sum_{i=1}^{n} \theta_{i}$ of a class is zero;

3) If $\left|C_{p}-C_{q}\right| \leq T b$, then these two types of classes will be merged. The cluster center is recalculated and the number of clusters is decreased by one.

4) The intra-class thresholds are changed according to equation (9), as soon as the number of clusters changes.

$$
\operatorname{Tin}=\left\{\begin{array}{c}
\frac{\pi}{6}, \text { if } \theta_{\max i}-\theta_{\min i} \leq \frac{\pi}{2} \\
\frac{\left|\theta_{\max i}-\theta_{\min i}\right|}{2}, \text { if } \quad \theta_{\max i}-\theta_{\min i}>\frac{\pi}{2}
\end{array}\right.
$$

5) Re-clustering according to the new parameters, until the cluster centers no longer change, otherwise, turn to the second step.

\section{EXPERIMENTAL RESULTS AND ANALYSIS}

The crowd test videos used in this paper come from UCF[12]. In order to avoid noise interference, the global optical flow field is obtained by the approach in [11]. The test results show in Fig.1, Where Fig.1 (a) and (b) are two adjacent frames of three different videos with size of $320 \times 240$ and Fig. 1 (c) is the optical flow field corresponding to the three videos. 

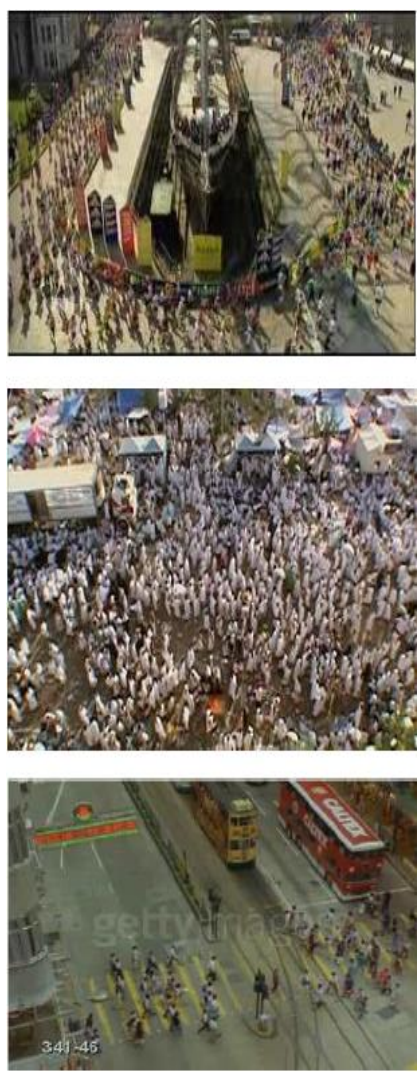

(a) The nth frame
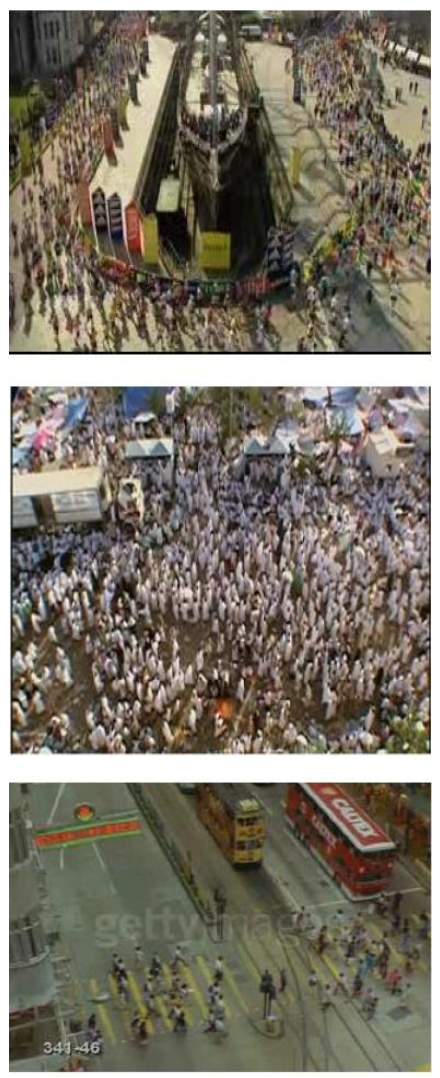

(b)The $\mathrm{N}+1$ th frame
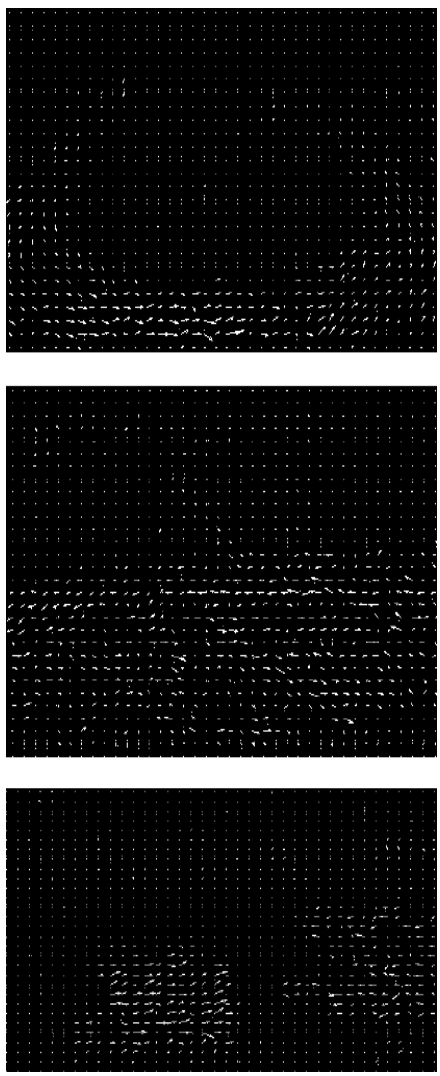

(c) Optical flow

Figure 1. Global optical flow calculation for different video sequences

Then, the motion flow segmentation of two videos in different scenes is performed by employing the algorithm proposed in [2] and in this paper. As shown in picture Fig. 2, where Fig. 2 (a) and (d) are test frame in two different crowd videos, Fig.2 (b) and (e) show the segmentation results depending on the method of [2], while the Fig.2 (c) and (f) are the segmentation effect by our algorithm. As can be seen from the Fig.2 (b) and 2 (c), the foreground motion flow is divided into one kind by the algorithm of literature[2], but the method used in this paper divides the motion flow into three types including horizontal right, vertical upward and vertical downward. Additionally ,the results in Fig.2 (e) and (f) show that the segmentation effect of the two methods is similar. 


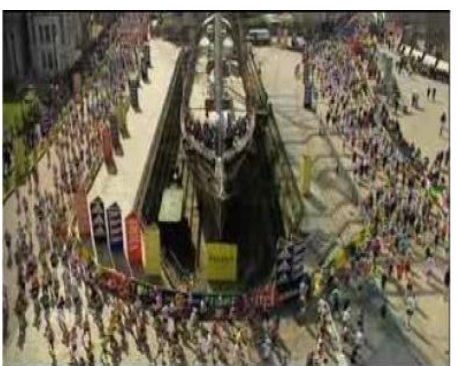

(a) Test frame

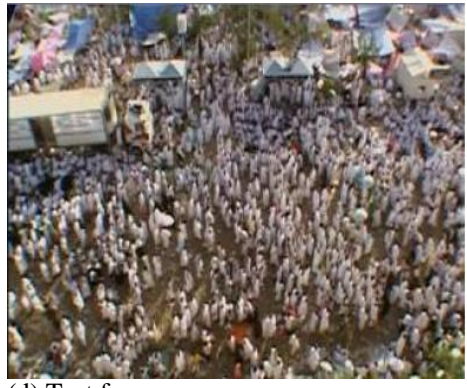

(d) Test frame

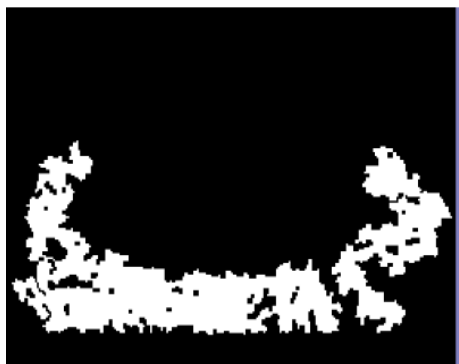

(b) Segmentation effect of [2]

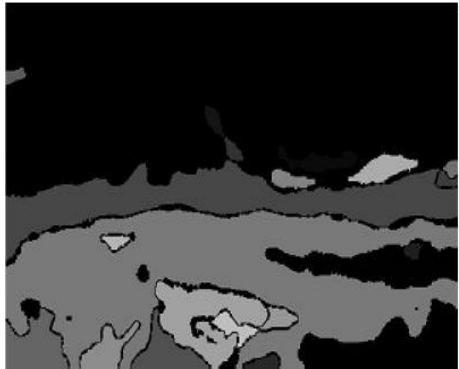

(e) Segmentation effect of [2]

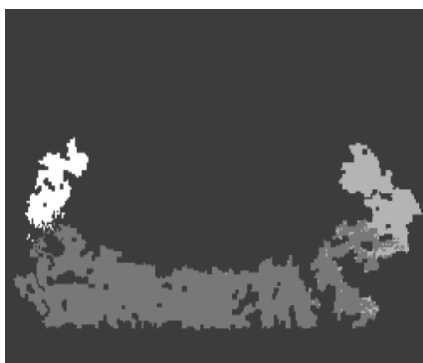

(c) Our algorithm

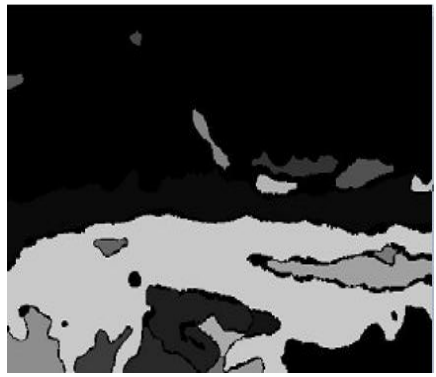

(f) Our algorithm

Figure 2. Comparison of motion flow segmentation results

TABLE I. COMPARISON THE AVERAGE ERROR OF MOTION FLOW SEGMENTATION

\begin{tabular}{|c|c|c|c|c|}
\hline & Frame & Real value & The average error of [2] & $\begin{array}{c}\text { The average error of } \\
\text { this paper }\end{array}$ \\
\hline Scene 1 & 440 & 3 & $20.5 \%$ & $15.2 \%$ \\
\hline Scene 1 & 67 & 4 & $14.6 \%$ & $13.8 \%$ \\
\hline
\end{tabular}

Experimental results showed, in a scene similar to that in Fig. 2 (a), the motion trajectories of the different directions are connected to each other. Additionally, form the table1 we can also know that the approach adopted in this paper is more accurate than the method in [2]. In other sequences similar to Fig.2 (d), motion trajectories of different directions are not connected. The results of this paper are basically the same as those of [2]. That is to say, our algorithm can be applied in a wider range of scenarios.

\section{CONCLUSION}

A motion flow segmentation algorithm has been proposed based on optical flow angle in this paper. The global optical flow was obtained by the method of reference [11] for obtaining a more accurate optical flow values. At the same time, the motion objects obtained by the theory of particle dynamics will be treated as the prospect mask. Then we can apply it to extract the optical flow information of foreground in global optical flow field, so that a good experiment results must be presented without the interference of optical flow values in the background. On this basis, the algorithm of dynamic K-means clustering is employed to cluster the optical flow angle. And finally it allows us to achieve the segmentation of crowd flow. The experiment results prove that the algorithm in this paper is closer to those actual scenes. Therefore our algorithm is great practical significance and application value for further crowd density estimation, target tracking and behavior understanding.

\section{ACKNOWLEDGMENTS}

This work was supported by the National Natural Science Foundation of China (61263034) and Youth science and technology talents growth projects in Guizhou province department of education (Qian education cooperation KY word [2016]165).

\section{REFERENCE}

[1] M. Aril and J. Richard, "Detecting Dominant Motions in Dense Crowd,"Signal Processing, 2008, 2(4): 568-581.

[2] S.Ali, and M. Shah, "A Largrangian Particle Dynamics Approach for Crowd Flow Segmentation and Stability Analysis,"International Conference on Computer Vision and Pattern Recognition, 2007: 1-6.

[3] A.C. Davies, J.H. Yin and S.A. Velastin, "Crowd monitoring using image processing,’IEE Electron. Commun. Eng. J.,1995, 7(1): 37-7.

[4] R. Ma, L. Li, W Huang, and Q. Tian, "On pixel count based crowd density estimation for visual surveillance,"in Proc. IEEE Conf. Cybernetics and Intelligent Systems, 2004, 1:170-173.

[5] T. Schlogl, B. Wachmann, H. Bischof, W Kropatsch, "People Counting in Complex Scenarios,"Vision with non-traditional sensors, Proc.of 26th Workshop of the Austrian Association for Pattern Recognition 2002, 160: 159-166.

[6] A.B Chan, Z. Liang, and N. Vasconcelos,"Privacy preserving crowd monitoring:Counting people without people models or trackin,"in 
Proc. IEEE Conf.Computer Vision and Pattern Recognition, 2008: 1-7.

[7]A.B. Chan and N. Vasconcelos,"Modeling, clustering, and segmenting video with mixtures of dnamic textures ,"IEEE Trans. on Pattern Analysis and Machine Intelligence, 2008, 30(5): 9-26.

[8]V.Rabaud and S. Belongie, "Counting crowded moving objects,"in Proc. IEEE Conf. Computer Vision and Pattern Recognition, 2006: 705-711.

[9]G J. Brostow and R. Cipolla,"Unsupervised Baayesian detection of independent motion in crowds,"in Proc. IEEE Conf. Computer Vision and Pattern Recognition, Washington, DC, 2006: 594--601.
[10]Guillaume Lapeyre,"Characterization of finite-time Lyapunoy exponents and vectors in two-dimensional turbulence,"Chaos, 2002, 12(3): 688-698.

[11]Papenberg N, Bruhn, Andr\&\#, Brox T, et al,'Highly Accurate Optic Flow Computation with Theoretically Justified Warping,'International Journal of Computer Vision, 2006, 67(2):141-158.

[12]http://www.cs.cmu.edu/ saada/Projects/CrowdSegmentation/index.htm 